Revue internationale P.M.E.

Économie et gestion de la petite et moyenne entreprise

\title{
Le regroupement des demandes de crédits des petites et moyennes entreprises tunisiennes dans le contexte d'asymétrie d'information
}

\section{Abdelwahed Omri et Meryem Bellouma}

Volume 17, numéro 2, 2004

URI : https://id.erudit.org/iderudit/1008457ar

DOI : https://doi.org/10.7202/1008457ar

Aller au sommaire du numéro

Éditeur(s)

Presses de l’Université du Québec

ISSN

0776-5436 (imprimé)

1918-9699 (numérique)

Découvrir la revue

Citer cet article

Omri, A. \& Bellouma, M. (2004). Le regroupement des demandes de crédits des petites et moyennes entreprises tunisiennes dans le contexte d'asymétrie d'information. Revue internationale P.M.E., 17(2), 43-63.

https://doi.org/10.7202/1008457ar
Résumé de l'article

Compte tenu de l'opacité de la structure informationnelle de la plupart des petites et moyennes entreprises solliciteuses d'un crédit, les banques commerciales tunisiennes ont une attitude frileuse à l'égard de leur financement. Après avoir recensé les motifs de cette posture méfiante, nous proposons de tester empiriquement la validité de la stratégie de regroupement, sur la base d'un échantillon composé de 523 dossiers de crédits de petites et moyennes entreprises tunisiennes.

Le test réalisé montre que la banque considérée ne tient pas compte du risque relatif à chaque entreprise lors de la détermination du taux d'intérêt, d'où une évidence à l'égard du recours à la stratégie de regroupement. Par ailleurs, le test révèle que la fixation du taux d'intérêt est tributaire de certaines modalités de garanties et du levier financier. 


\title{
Le regroupement des demandes de crédits des petites et moyennes entreprises tunisiennes dans le contexte d'asymétrie d'information
}

\author{
Abdelwahed OMRI \\ Faculté des sciences économiques et de gestion de Tunis
}

Meryem BELLOUMA

Institut supérieur de gestion de Tunis

\section{MOTS CLÉS}

\section{Asymétrie informationnelle - Décision de crédit \\ Stratégie de regroupement \\ Petites et moyennes entreprises tunisiennes}

\begin{abstract}
RÉSUMÉ
Compte tenu de l'opacité de la structure informationnelle de la plupart des petites et moyennes entreprises solliciteuses d'un crédit, les banques commerciales tunisiennes ont une attitude frileuse à l'égard de leur financement. Après avoir recensé les motifs de cette posture méfiante, nous proposons de tester empiriquement la validité de la stratégie de regroupement, sur la base d'un échantillon composé de 523 dossiers de crédits de petites et moyennes entreprises tunisiennes.
\end{abstract}

\section{LES AUTEURS}

ABDELWAHED OMRI est maître de conférences à la Faculté des sciences économiques et de gestion de Tunis et directeur de recherche à l'unité de recherche ETHICS. Ses travaux de recherche portent sur les rôles de l'information financière, la Bourse, les banques et la corporate governance. II a publié de nombreux articles dans des revues françaises et internationales. II est l'auteur d'un ouvrage intitulé Gestion financière, édité par L'Harmattan, 2003. Adresse : Faculté des sciences économiques et de gestion de Tunis, B.P. 248, Manar II, Tunisie. Courriel : <abomri@yahoo.fr>.

MERYEM BELLOUMA est doctorante et chercheure à l'Institut supérieur de gestion de Tunis et membre de l'unité de recherche ETHICS. Ses recherches portent sur le domaine bancaire et sur les relations contractuelles de crédits. Elle s'intéresse également à la performance et à la corporate governance. Adresse : Institut supérieur de gestion de Tunis, 45, rue de la Liberté, cité Bouchoucha, 2000 Bardo, Tunisie. Courriel : <mbellouma@yahoo.fr>. 
Le test réalisé montre que la banque considérée ne tient pas compte du risque relatif à chaque entreprise lors de la détermination du taux d'intérêt, d'où une évidence à l'égard du recours à la stratégie de regroupement. Par ailleurs, le test révèle que la fixation du taux d'intérêt est tributaire de certaines modalités de garanties et du levier financier.

\begin{abstract}
Most of the firms have a less developed informational system. Banks have so, in theory, a repugnant attitude regarding to their financing. The purpose of this work is to establish if the bank strategies in fixing the credit conditions is the pooling one. In order to do this, we use a sample of 523 credit files of small and medium Tunisian businesses.

The empirical test realized seems to indicate that the considered bank uses pooling contracts, and it is showned that the charged rates do not represent correctly the future risk of the borrowers. In addition, the test reveals that the interest rate is dependent on some modalities of guarantees and the financial leverage.
\end{abstract}

\title{
RESUMEN
}

La mayor de las firmas han un sistema di información menos desarrollado. Bancos han, en consecuencia, una actitud friolera con respecto al suyo financiamiento. El propósito de este trabajo es testar empíricamente la validez della estrategia del reagrupamiento, teniendo como base una muestra compuesto de 523 respaldos dos créditos de las escasas medias sociedades Tunecinas.

La prueba empírica afemina do muestra aquella banca no tena consideración el riesgo relativa a cada sociedad in momento las determinación de tipo de interés. Donde una evidencia con respecto del recurso allá estrategia del reagrupamiento. Alo obstante, la prueba empírica revela cuela fijación del tipo de interés es dependiente de ciertos modalidad de garantías o de la palancada financiera.

\section{ZUSAMMENFASSUNG}

Aufgrund der Undurchsichtigkeit der Informationsstruktur einer Mehrzahl der kleinen und mittleren Unternehmen, die Kreditanträge stellen, zeigen sich die tunesischen Geschäftsbanken zurückhaltend und vorsichtig bei deren Finanzierung. Nachdem wir die Gründe dieser eher misstrauischen Grundhaltung aufgezählt haben, wollen wir die Gültigkeit der strategischen Neusegmentierung mittels einer Stichprobe von 523 Kreditakte von KMUs testen.

Ein durchgeführter Test zeigt, dass die kreditsprechenden Banken das unternehmensspezifische Risiko des Antragstellers bei der Festlegung des Zinssatzes nicht berücksichtigen. Die Notwendigkeit einer strategischen Neusegmentierung ist dadurch hinreichend begründet. Der Test brachte auch hervor, dass die Bestimmung des Zinssatzes von Garantiemodalitäten und von der Finanzierungsform abhängig ist. 


\section{Introduction}

Les PME constituent l'élément essentiel du maillage économique de la Tunisie ; elles représentent un moteur de croissance et d'emploi puissant. Par ailleurs, l'économie tunisienne est encore très largement une économie d'endettement, de surcrôt essentiellement assurée par le secteur bancaire. Cette évidence laisse présager que la source primordiale de financement des PME tunisiennes est le crédit bancaire.

L'endettement total s'élevait à la fin de l'année 2001 à 45 milliards de dinars tunisiens (TND) ${ }^{1}$, soit $145 \%$ du PIB, et l'endettement intérieur comptait pour les deux tiers de ce montant. Sur cette enveloppe, $85 \%$ ont été fournis par les banques, la partie prépondérante étant attribuée à des PME (Myard et Alperte, 2003).

Ainsi, en Tunisie, c'est du secteur bancaire que part le financement de l'économie (composée essentiellement par des petites et moyennes entreprises) pour irriguer ses activités et entretenir son expansion, étant donné que la Bourse des valeurs mobilières de Tunis contribue très peu au financement des entreprises.

Toutefois, malgré l'importance financière de leurs liens d'affaires, les relations entre les banques et les entreprises tunisiennes sont souvent complexes. En effet, le banquier est soumis dans sa relation avec les petites et moyennes entreprises à de nombreuses sources d'asymétries d'information.

Cette disparité informationnelle s'explique par la fragilité du système d'information des PME tunisiennes. De même, la fluctuation de la conjoncture économique influence les parts de marchés des PME. À cela s'ajoute le fait que les banquiers tunisiens se plaignent de l'inflexibilité de la gestion des dirigeants des petites et moyennes entreprises qui, dans la majorité des cas, s'occupent à la fois des problèmes de la politique générale de l'entreprise et des questions opérationnelles.

Ainsi, si l'asymétrie informationnelle se révèle être une hypothèse vérifiable autour de laquelle des théories financières cohérentes peuvent être construites, la banque commerciale tunisienne est amenée à modifier radicalement ses procédures de suivi du risque de crédit et à renouveler ses outils, ses instruments de gestion et ses modèles de prise de décision. Par ailleurs, la banque tunisienne doit tirer des leçons de ses expériences de prêt et envisager des solutions pour atténuer le risque auquel elle s'expose.

Plus précisément, la banque commerciale tunisienne doit instaurer une gestion adaptée aux diverses mutations environnementales imposées par la prolifération de la concurrence et la fin du protectionnisme. En d'autres termes, elle est appelée

1. $\mathrm{TND}=1,4 \$ \mathrm{US}$.

Revue internationale P.M.E., vol. 17, nº 2, 2004 
à adapter son processus d'octroi de crédit aux spécificités de sa clientèle, à la nature des informations mises à sa disposition et au néo-paysage économique ouvert et mouvementé (Omri et Bellouma, 2003).

De ce fait, nous proposons dans cette étude de voir le degré d'adaptabilité de la stratégie de regroupement par la banque tunisienne. En d'autres termes, nous essayerons de vérifier si la banque tunisienne, face à une clientèle hétérogène de PME, offre un contrat de crédit indépendant du risque relatif à chaque entreprise, choix imposé par l'impossibilité de segmenter la clientèle.

Cette étude est motivée par l'insuffisance des recherches empiriques sur la décision de l'analyste de crédit en s'appuyant sur son input informationnel. De même, nous nous proposons de combler un certain manque de la littérature actuelle, car malgré les multiples études publiées dans le domaine du financement des PME, le cas tunisien demeure non étudié.

Pour ce faire, nous recensons, en premier lieu, les motifs de l'attitude frileuse de la banque commerciale à l'égard du financement des petites et moyennes entreprises. En deuxième lieu, nous essayerons de présenter la notion de la stratégie de regroupement en exposant sa légitimité. Nous essayerons par la suite de cerner le degré de validité de la stratégie de regroupement dans l'explication du comportement du banquier tunisien, cherchant à réduire l'impact de la disparité informationnelle.

\section{L'interface entre les PME et les banques: le point de vue des banques}

La théorie d'intermédiation financière s'est basée sur l'imperfection du marché et la remise en cause de l'hypothèse afférente à la disponibilité équitable de l'information pour tous les intervenants. En effet, l'existence des informations asymétriques a engendré la réorientation des différentes études, notamment dans le domaine bancaire en matière d'octroi de crédits où les relations contractuelles sont généralement caractérisées par la répugnance de la partie qui dispose d'informations privées (l'emprunteur) de les révéler.

Ainsi, l'importance de l'obstacle financier à la création et au développement des petites et moyennes entreprises est aujourd'hui au cœur de nombreux débats. Ces dernières, considérées comme l'instrument de relance de toute économie industrialisée ou émergente, comme c'est le cas de la Tunisie, sont sujettes à une limitation de crédit sur le marché bancaire. En effet, il est incontestable que la difficulté majeure à laquelle font face les banques commerciales réside dans leur incapacité à cerner d'une façon suffisamment précise le risque présenté par les clients et l'incertitude de leur prospérité. 
Par ailleurs, l'existence des problèmes de communication et de conflit d'intérêts entre les dirigeants de PME et les responsables des banques nécessite la conciliation entre deux rationalités différentes. Pour être plus précis, on peut dire que les «préoccupations sous-optimales» du dirigeant de PME se heurtent à la politique optimaliste du prêteur (Colot et Michel, 1996).

Toutefois, comme le préconise la théorie de l'agence, le créancier peut engager des dépenses de contrôle limitant le comportement opportuniste des emprunteurs. En effet, une fois le crédit accordé, le dirigeant de la PME peut substituer le projet initial par un autre plus risqué afin d'augmenter son rendement ou, encore, adopter une politique d'investissement sous-optimale (le sous-investissement) en utilisant une partie des fonds prêtés pour ses besoins personnels (achat d'une voiture, voyages, etc.).

À cet égard, Jensen et Meckling (1976) avancent que les créanciers, en anticipant de telles déviations de comportement, intègrent des mécanismes de contrôle dans le contrat de prêt (des clauses limitant les actions de l'entreprise en matière d'investissement ou restreignant son accès au cash-flow engendré et la distribution de dividendes, etc.). Les auteurs relèvent aussi que le dirigeant de l'entreprise serait amené, à son tour, à assumer des coûts d'agence pour montrer à sa banque qu'il n'agit pas à l'encontre de son intérêt (production des états financiers, etc.).

Outre l'antagonisme entre fonctions et objectifs, la banque est réticente à financer cette catégorie d'entreprises, dépourvues d'historique économique et financier (Colot et Michel, 1996). Dans cette perspective, l'octroi d'un crédit à une PME implique un surplus de risque par rapport à la même opération avec une grande entreprise. Plus précisément, Berryman (1983) et Peacock (1985) estiment que la méfiance des banquiers à l'égard des demandes de crédits des PME résulte de leurs éventuelles carences managériales. Également, l'insuffisance de la formation financière et le peu d'intérêt porté à la planification sont fréquemment évoqués par les banquiers pour expliquer la difficulté de leurs relations avec les PME (Bissiriou, 1989; Wtterwulghe et Janssen, 1995).

Un autre aspect susceptible d'amplifier le risque et l'incertitude que présentent les petites et moyennes entreprises tunisiennes consiste dans la fragilité de leur système informationnel. Les dirigeants hésitent à fournir des informations fiables sur leurs entreprises, que ce soit pour des raisons fiscales, concurrentielles ou liées aux coûts de leur production.

À cet égard, il y a lieu de se pencher sur la théorie de signalisation voulant que les dirigeants soient bien informés (sur les perspectives financières de leur entreprise) par rapport à d'autres partenaires, principalement les banques. Ainsi, les agents mieux informés (les dirigeants) vont chercher à transmettre aux créanciers 
(les banques) un message relatif à la nature de la firme qu'ils gèrent. Par conséquent, l'un des moyens de signalisation relevés dans les travaux en économie est la structure financière de la firme qui renseigne sur son niveau de risque, donc, sur sa capacité à honorer ses engagements. Ainsi, à travers son niveau d'endettement, l'emprunteur signale sa qualité au créancier et son aptitude à assumer ses obligations (Ross, 1977).

Pour Leland et Pyle (1977), la structure de propriété de la firme revêt également une importance particulière pour le prêteur. En effet, l'implication du dirigeant dans le capital de l'entreprise, selon ces auteurs, apparaît corrélée positivement avec la valeur de la firme. Ainsi, un capital familial a de fortes chances de se traduire par une politique d'investissement plus conservatrice et donc moins risquée pour les banques dans la mesure où la même famille supporte l'ensemble des risques.

Finalement, la littérature financière souligne l'importance des mécanismes incitatifs où la partie non informée, la banque, propose à la partie informée, le dirigeant de l'entreprise, un ensemble de contrats parmi lesquels les différents types d'agents (agents risqués versus agents sains) pourront choisir en fonction de leurs caractéristiques. Les contrats révélateurs les plus usuels reposent ainsi sur la fixation du niveau du taux d'intérêt et des garanties (Bester, 1985; Besanko et Thakor, 1987). Selon ces modèles, les entreprises peu risquées n'acceptent pas de s'endetter à un coût jugé prohibitif, mais elles sont prêtes à apporter en garantie un montant élevé d'actifs. À ce niveau, il y a lieu de cerner la politique de la banque commerciale tunisienne en matière de fixation du taux d'intérêt ; mais auparavent, nous allons jeter un peu de lumière sur la stratégie de regroupement qui représente une forme de limitation du risque de crédit.

\section{La légitimité de la stratégie de regroupement dans un contexte d'information asymétrique}

La distorsion entre l'information dont dispose le dirigeant et celle qu'il met à la disposition de son interlocuteur bancaire conduit la banque commerciale à fixer, a priori, un niveau de risque élevé à toute opération de crédit ; une telle surestimation l'amène à exiger des taux d'intérêt trop importants (Fernando et Mallick, 2002).

Stiglitz et Weiss (1981) ont montré que la banque n'a pas intérêt à agir de la sorte; en effet, si l'augmentation du taux débiteur permet un ajustement de l'offre et de la demande, les emprunteurs peuvent répondre à cette croissance par une amplification du risque des projets entrepris. Par ailleurs, certaines entreprises non risquées, incapables de supporter des taux débiteurs élevés, peuvent être amenées à renoncer à leurs investissements (Berger et al., 2002). La banque serait alors 
exposée à une baisse de son rendement anticipé. C'est pourquoi l'asymétrie d'information entre la banque et les emprunteurs sur le marché du crédit est à l'origine du rationnement (Psillaki, 1995; Berger et Udell, 2002). Selon cette théorie, les petites et moyennes entreprises sont particulièrement sujettes au rationnement. En effet, les entreprises exclues sont tout d'abord celles dont la rentabilité attendue est la plus faible, donc les plus risquées (Ang, 1991; Black et Strahan, 2001 ; Bodt, Lobez et Statnik, 2000).

En cas d'information parfaite, la banque est à même de contrôler et de réduire, voire d'anéantir le risque de crédit. En outre, la population d'emprunteurs potentiels pourrait être divisée en plusieurs groupes d'individus différenciés sur la base d'un critère de risque. Toutefois, l'importance de l'asymétrie d'information entre les banques et les PME ne permet pas de distinguer convenablement le type des entreprises solliciteuses de crédit (Scott, 2003). Selon Stiglitz et Weiss (1981) et Lehmann et Neuberger (2002), on ne peut remédier à cette situation en réalisant des économies d'échelle grâce au regroupement des demandes de crédits de plusieurs PME, d'où la notion de la stratégie de regroupement.

La stratégie de regroupement consiste à recourir à un contrat indépendant du risque relatif à chaque entreprise ; elle est adoptée par la banque lorsqu'il lui est impossible de segmenter sa clientèle. Cela découle de l'hétérogénéité que présentent les emprunteurs sur le plan de leurs opportunités d'investissement qui n'est discernable que dans une perspective ex post de l'accord du crédit (James, 1987). De même, elle peut être utilisée par celle qui préfère minimiser les coûts de gestion engendrés par la considération de chaque dossier de crédit comme un cas spécifique, ce qui les amène à opter pour une gestion globale du risque (Bodt, Lobez et Statnik, 2000). Cette constatation laisse présager que le coût substantiel, découlant de la création de contrats de crédit sur mesure, est loin d'être couvert par le gain éventuel résultant d'une étude minutieuse de chaque perspective de la demande du client et de son risque éventuel.

En d'autres termes, la stratégie de regroupement suppose que le taux d'intérêt demandé par la banque est indépendant du risque particulier de l'entreprise ; bien qu'il reflète le risque moyen des emprunteurs, ce dernier est considéré comme une unité autonome de l'ensemble (Bodt, Lobez et Statnik, 2000). Par conséquent, la banque offre un contrat de crédit, caractérisé par un seul critère de discrimination ou encore de segmentation de la clientèle, à savoir le taux d'intérêt (Stiglitz et Weiss, 1981).

Les adeptes de cette stratégie soutiennent que la banque doit réduire le risque global de son portefeuille de crédits en optant pour des combinaisons optimales des différentes demandes éventuelles, sans chercher à minimiser le risque individuel que présente chaque client potentiel (Bodt, Lobez et Statnik, 2000). 
Rappelons ici que cette étude cherche à vérifier le degré d'application du regroupement des demandes de crédits dans le contexte bancaire tunisien. Nous proposons alors un modèle économétrique formulant la stratégie de regroupement afin de cerner sa validité.

\section{4. Évidences empiriques sur l'utilisation de la stratégie de regroupement par la banque tunisienne}

L'objectif de cette étude est de tester empiriquement l'hypothèse issue de l'analyse théorique et selon laquelle la banque commerciale adopte la stratégie de regroupement en matière d'octroi de crédits. Nous présentons l'échantillon d'entreprises retenu, puis la mesure des variables utilisées ainsi que les résultats obtenus du modèle testé.

\subsection{Données et constitution de l'échantillon}

Les données utilisées dans l'étude proviennent de l'ensemble des relations de crédits entretenues par une banque commerciale tunisienne durant la période s'étalant de 1998 à 2001, à savoir un nombre de dossiers s'élevant à 523. Il s'agit principalement de données annuelles (observées au 31 décembre des différentes années de l'étude) tirées des états financiers des entreprises retenues.

Pour constituer notre échantillon, nous avons utilisé les critères de sélection suivants :

- Les entreprises retenues dans le cadre de cette étude sont des petites et moyennes entreprises ayant un effectif inférieur à 500 employés.

Définir cette catégorie d'entreprises n'est pas une chose aisée, vu sa grande hétérogénéité découlant de la coexistence de toutes petites entreprises, de petites et de moyennes qui se différencient par des modes de propriété, d'évolution et un environnement qui leur est spécifique. La définition de cette population varie ainsi en fonction de l'objet de l'étude et des données disponibles. Le rapport de Bolton (1971), qui fut à l'origine de la reconsidération des PME par l'économie industrielle en Angleterre, retient trois critères essentiellement qualitatifs : une PME détient une part relativement petite de son marché ; elle est gérée de manière personnelle par son propriétaire ; elle est indépendante, c'est-à-dire non intégrée dans une grande structure et ses propriétaires prennent leurs décisions sans contrôle extérieur.

- Dans de nombreux pays, le concept du nombre de salariés a été préféré pour définir les entreprises de cette catégorie. Ce critère simple à calculer 
et facile d'accès présente une bonne corrélation avec la valeur ajoutée et trouve par ailleurs, dans le contexte tunisien, une résonance politique évidente (il permet de voir si cette population d'entreprises contribue de façon significative à la création d'emplois).

Les entreprises appartenant aux secteurs regroupant les institutions financières, les assurances et les organismes publics ont été exclus.

\subsection{Variables retenues dans l'analyse}

Cette sous-section recense les variables à incorporer dans le modèle assigné à l'hypothèse formulée préalablement. Nous présentons les variables figurant au sein du contrat du crédit, la variable décisionnelle (le risque) et les autres variables explicatives.

\subsubsection{Les variables contractuelles}

Il s'agit des variables figurant dans le contrat de crédit, à savoir le taux d'intérêt et les garanties exigées.

INTER. Le taux d'intérêt présente la variable à expliquer. Il s'agit du taux du marché monétaire (TMM) majoré de la marge de la banque. Il constitue un élément déterminant du contrat de crédit et doit faire l'objet du consentement du client et de la banque puisque son choix doit être viable pour l'entreprise et cohérent avec ses spécificités (Duffee, 1998; Cossin et Hricko, 2001). La vérification d'un tel ajustement permet, entre autres, d'éviter le comportement opportuniste de l'emprunteur qui peut manquer à ses engagements en raison d'un taux d'intérêt prohibitif (Petersen et Rajan, 1994 ; Nakamura, 1998).

PLGAR. La modalité «plusieurs garanties» prend la valeur 1 si le contrat inclut plusieurs garanties (hypothèques, nantissement, cautions solidaires, etc.) et 0 ailleurs. L'emprunteur offre une panoplie de garanties dans le but de bénéficier d'un taux d'intérêt avantageux.

CASOL. La modalité «cautions solidaires » prend la valeur 1 si le contrat inclut la caution solidaire du dirigeant de l'entreprise et 0 ailleurs. L'acceptation du dirigeant d'une clause prévoyant une caution solidaire serait perçue par la banque comme un signal positif, puisqu'il accepte d'engager sa responsabilité personnelle dans les décisions financières de l'entreprise (Avery, Bostic et Samolyk, 1998).

NANTS. La modalité «nantissement» prend la valeur 1 si le contrat inclut un nantissement et 0 ailleurs. Cette modalité de garanties qui porte sur les stocks ou les créances clients permet à la banque de suivre continuellement la gestion courante de l'emprunteur. 
HYPOT. La modalité «hypothèque» prend la valeur 1 si le contrat inclut une hypothèque et 0 ailleurs. L'hypothèque permet de signaler l'absence du risque moral de l'emprunteur, du fait qu'il ne serait pas amené à s'engager dans des comportements opportunistes au détriment de l'intérêt de la banque.

SSGAR. La modalité «sans garantie » prend la valeur 1 si le contrat n'inclut pas de garanties et 0 ailleurs. Cette modalité est considérée comme étant la modalité de base.

\subsubsection{La variable décisionnelle}

RISQ. La variable « risque » est mesurée par le différentiel entre le cash-flow du début de la période d'analyse et son minimum enregistré tout au long de la durée de l'étude, rapporté au total de fonds propres de l'entreprise pendant la première année retenue. Ce rapport met en exergue le risque d'insolvabilité et l'incapacité de l'entreprise à honorer ses engagements.

Nous considérons que l'asymétrie informationnelle provient essentiellement du risque opérationnel de l'entreprise. Cette variable est supposée inconnue de la part de la banque commerciale et présente une donnée essentielle pour estimer la prime de risque à imputer dans le taux d'intérêt à charger (Merton, 1974).

Si l'hypothèse afférente à l'adoption de la stratégie de regroupement est vérifiée, nous nous attendons à ce que la variable risque ne soit pas significative ; car, dans le cadre de cette stratégie, la banque prend en considération le risque global de sa clientèle et non pas le risque spécifique à chaque entreprise.

\subsubsection{Les variables explicatives}

F.JURID. La forme juridique de l'entreprise peut être mesurée par une variable auxiliaire égale à 1 si la firme est une société anonyme (SA) et 0 si elle est une société à responsabilité limitée (SARL). Cette modalité (SARL) est considérée comme étant la modalité de base ; elle détermine la responsabilité des actionnaires et des dirigeants en imposant des contraintes spécifiques sur les capitaux propres de l'entreprise.

Par ailleurs, elle affecte le montant d'informations privées détenues par les dirigeants, leur incitation et leur capacité à déplacer le risque vers la banque. En effet, les sociétés anonymes et les sociétés à responsabilité limitée restreignent l'accès de la banque aux actifs privés des propriétaires en cas de non-respect des engagements conclus. C'est pourquoi le risque de crédit tend à être plus élevé pour ce type d'entreprises (Elsas et Krahnen, 1998). 
LEVER. Le levier financier est mesuré par le ratio du total des dettes sur la valeur comptable totale de l'entreprise. Ainsi, un niveau d'endettement élevé sera associé à un risque important de défaillance.

SP.ACTF. Concernant la mesure de la spécificité de l'actif, nous retenons le ratio de l'actif non courant de l'entreprise à l'actif total. Ce critère apparaît comme une variable de contrôle additionnelle, devant être prise en considération par la banque commerciale dans son analyse pour déterminer l'octroi de crédits. La spécificité de l'actif crée des problèmes pour l'octroi de crédits, étant donné que les caractéristiques de non-redéploiement de l'actif, trop spécifiques, altèrent la capacité de la banque à le liquider en cas de non-remboursement.

SECT.EC. Le secteur d'activité dans le cadre de cette étude est considérée comme une variable muette, elle prend la valeur 1 si l'entreprise appartient au secteur industriel et la valeur 0 pour le secteur des services. Cette modalité (service) est considérée comme étant la modalité de base. L'activité de l'entreprise dépend de la conjoncture économique et des différents changements de l'environnement. Chaque secteur a son propre cycle d'activité et présente des menaces et des opportunités distinctes suscitant des réactions différentes au regard de leur ampleur et de leur synchronisation. Cette variable est objectivement connue de la part de la banque et peut l'orienter dans sa prise de décision en matière d'octroi de crédits.

CLIENT. La variable «relation client» sera mesurée par le ratio rapportant les dettes bancaires à moyen et long terme au total des dettes bancaires de l'entreprise. D'une manière générale, la banque qui a une certaine confiance envers un client particulier peut aisément accepter une relation à long terme et s'épargner les fréquentes négociations qu'exigent les crédits à court terme.

Certaines caractéristiques de l'échantillon peuvent être dévoilées. En effet, le panel est majoritairement composé de sociétés à responsabilité limitée (en moyenne, durant les cinq périodes de l'étude, elles présentent $63 \%$ des entreprises incluses dans l'échantillon). En outre, une grande proportion des entreprises incluses dans l'analyse (en moyenne $95 \%$ ) œuvrent dans le secteur des services, contre une minorité d'entreprises exerçant une activité industrielle. Avant de nous pencher sur les résultats de l'étude, nous analyserons quelques statistiques descriptives des variables quantitatives rapportées dans le tableau 2. 


\section{TABLEAU 1}

Présentations et mesures des variables retenues

\begin{tabular}{|c|c|c|}
\hline Variable & Symboles & Indicateurs de mesure \\
\hline Taux d'intérêt & INTER & Le taux du marché monétaire $(\mathrm{TMM})$ + la marge de la banque \\
\hline Risque & RISQ & $\begin{array}{l}\text { Cash-flow (début de la période) - min (les cash-flows) / fonds } \\
\text { propres (début de la période) }\end{array}$ \\
\hline Forme juridique & F.JURID & S.A. : 1 , sinon 0 (SARL: modalité de base) \\
\hline Levier financier & LEVER & $\begin{array}{l}\text { Valeur comptable totale des dettes de l'entreprise / valeur } \\
\text { comptable de l'entreprise totale }\end{array}$ \\
\hline Spécificité de l'actif & SP.ACTF & Actif non courant / actif total \\
\hline Activité & SECT.EC & Industrie : 1 , sinon 0 (Service: modalité de base) \\
\hline \multirow[t]{2}{*}{ Garantie } & $\begin{array}{l}\text { PLGAR } \\
\text { CASOL } \\
\text { NANTS } \\
\text { HYPOT }\end{array}$ & $\begin{array}{l}\text { Plusieurs garanties : } 1 \text {, sinon } 0 \\
\text { Cautions solidaires : } 1 \text {, sinon } 0 \\
\text { Nantissement : } 1 \text {, sinon } 0 \\
\text { Hypothèque }: 1 \text {, sinon } 0\end{array}$ \\
\hline & SSGAR & Sans garantie: modalité de base \\
\hline Client & CLIENT & Dettes bancaires à moyen et long terme / dettes bancaires totales \\
\hline
\end{tabular}

\section{TABLEAU 2}

Statistiques descriptives des variables quantitatives

\begin{tabular}{lcccc}
\hline Variables & N & Moyenne & Écart type & Signification \\
\hline Client (CLIENT) & 523 & 0,314 & 0,288 & $0,000^{*}$ \\
Risque (RSQ) & 523 & 0,347 & 0,443 & $0,000^{*}$ \\
Taux d'intérêt (INTER) & 523 & 0,402 & 0,988 & $0,000^{*}$ \\
Spécificité de l'actif (SP.ACTF) & 523 & 0,208 & 0,316 & $0,000^{*}$ \\
Levier financier (LEVER) & 523 & 1,127 & 29,857 & $0,000^{*}$ \\
\hline
\end{tabular}

* Signification $<1 \%$.

La distribution moyenne de la variable afférente à la relation de clientèle (CLIENT) est de 0,314. L'étude de la variabilité met en évidence que le niveau de la relation client entretenue par les entreprises étudiées est variable.

Le taux moyen de risque (RISQ) de 34,7\% nous renseigne sur le degré de risque des entreprises tunisiennes incluses dans notre échantillon; il concorde avec l'énoncé relevé plus haut voulant que l'opacité informationnelle caractérise les emprunteurs solliciteurs de crédit.

Toutefois, l'observation du levier financier (LEVER) montre que la majorité des entreprises étudiées sont fortement endettées. En effet, le niveau moyen du levier est de $1,127 \%$. De même, on relève que la dispersion de la maturité des 
dettes autour de la moyenne est plus intense que celles des autres paramètres. Cette constatation implique que l'étendue de l'intervalle de variation de ce facteur est importante, c'est-à-dire que certaines entreprises contractent des crédits à très court terme, alors que d'autres prennent des engagements à long terme.

\subsection{Spécification du test empirique et résultats}

La validité de l'hypothèse afférente à la stratégie de regroupement est testée moyennant un modèle de régression multiple en données de panels. Formellement, il s'agit de régresser le taux d'intérêt (INTER) sur l'ensemble des variables énumérées ci-dessus. Pour en avoir une vision claire, nous procédons à deux régressions en introduisant et retirant alternativement les variables (CLIENT et LEVER) de manière à montrer leurs importances relatives sur le panel retenu et d'éviter le problème d'interdépendance qui surgit du fait que les dettes de long terme interviennent dans les deux variables de l'analyse. La formulation de la première régression donne le modèle économétrique suivant:

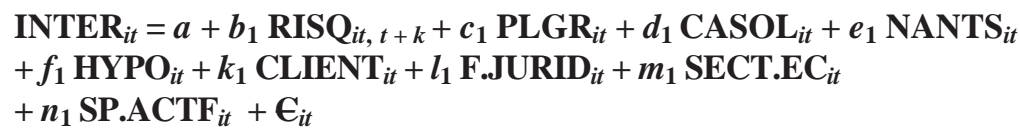

La variable à expliquer et les variables explicatives s'écrivent en fonction de deux indices $i$ et $t$, où $i$ est l'indice du dossier de crédit et $t$ celui de la période. Étant donné que les décisions de la banque sont prises à la date $t$ indépendamment ou non du risque économique, ce dernier peut être révélé à la date $t$ ou décalé à une date $t+k$.

\section{TABLEAU 3}

Estimations des paramètres relatifs au modèle (1)

Variable dépendante : taux d'intérêt (INTER)

\begin{tabular}{lccc}
\hline Variables & Coefficients & $\boldsymbol{t}$ & Signification \\
\hline RISQ & $0,656 \mathrm{E}-03$ & 1,114 & 0,265 \\
[PLGAR] & $0,189 \mathrm{E}-03$ & $-1,331$ & 0,183 \\
[CASOL] & $-0,942 \mathrm{E}-03$ & $-1,915$ & $0,055^{* *}$ \\
[NANTS] & $-0,002$ & $-2,009$ & $0,044^{*}$ \\
[HYPOT] & $-0,168 \mathrm{E}-03$ & $-1,862$ & $0,063^{* *}$ \\
CLIENT & $-7,454 \mathrm{E}-05$ & $-0,571$ & 0,568 \\
[F.JURID= SA] & 0,105 & 0,185 & 0,852 \\
[SECT.EC= Industrie] & $0,197 \mathrm{E}-02$ & 0,279 & 0,780 \\
SP.ACTF & 0,106 & 0,642 & 0,522 \\
\hline
\end{tabular}

** Signification $<10 \% *$ Signification $<5 \% \mathrm{R}^{2}=0,499\left(\mathrm{R}^{2}\right.$ ajusté $\left.=0,442\right)$. 
Les résultats de l'analyse révèlent que la banque retenue ne détermine pas le taux d'intérêt à accorder aux entreprises solliciteuses de crédit sur la base de leur risque, puisque le coefficient associé à la variable risque n'apparaît pas comme significativement différent de zéro.

Ainsi, avec une clientèle hétérogène composée de PME, la banque ne fixe pas le taux d'intérêt accordé en se basant sur le risque de chaque entreprise solliciteuse de crédit. Cette constatation prouve le recours de la banque retenue au regroupement des demandes de crédit. Cette stratégie permet de minimiser le risque global du portefeuille du crédit de la banque, étant donné que le segment des PME possède un trait commun, malgré les différences qui les caractérisent (occasion d'investissement, croissance, etc.). Ce trait commun, déjà soulevé dans la partie théorique, n'est autre que l'accentuation du risque de cette catégorie de clientèle, d'une manière indépendante des perspectives financières des entreprises à cause de l'opacité informationnelle qui les entache.

En observant la signification des différentes modalités de garanties retenues dans l'analyse, le tableau 3 montre que la caution solidaire, l'hypothèque et le nantissement présentent un élément explicatif du taux chargé par la banque. Les trois modalités varient d'une manière inversement proportionnelle avec la variable dépendante. Ce résultat reflète le postulat de la théorie afférente aux mécanismes d'incitations qui attribue un niveau de risque moral faible aux emprunteurs qui mettent à la disposition de leur banque des garanties. Et, par conséquent, le dirigeant de l'entreprise attend en contrepartie que la banque lui charge un taux d'intérêt relativement moins élevé (Bester, 1985). Ainsi, une entreprise, en mettant à la disposition de sa banque des garanties solides et diversifiées, est susceptible d'obtenir un crédit à un taux faible. À plus forte raison s'il y a une certaine stabilité dans la relation bancaire attestant de la cohérence de son comportement; en somme, l'augmentation du niveau de garantie explique la diminution du taux d'intérêt.

Toujours pour les garanties, la modalité «plusieurs garanties» (PLGAR) ne constitue pas une variable déterminante du taux d'intérêt, bien qu'elle présente le signe attendu (négativement corrélée avec le taux d'intérêt). La non-signifiance du coefficient associé à la modalité « plusieurs garanties » trouve sa légitimité dans le fait que l'échantillon de PME tunisiennes retenues dispose de peu d'éléments d'actif à mettre en garanties, ce qui peut d'ailleurs expliquer l'attitude frileuse des banques quant à leur financement.

Concernant le pouvoir explicatif de la variable afférente à la relation de clientèle (CLIENT), l'observation de la valeur de Student indique qu'elle n'a aucun effet sur la variable dépendante de l'analyse. Cette constatation s'accorde avec le principe de la stratégie de regroupement qui suppose que la banque traite sa clientèle indépendamment de sa spécificité et ne vise que la minimisation du risque global de son portefeuille. Ce résultat rejoint celui de Lehmann et Neuberger (2002) 
qui ont soutenu que la variable (CLIENT) n'a aucun effet sur le taux d'intérêt. Il contredit, en revanche, la thèse de Diamond (1991), qui a avancé que les entreprises ayant une relation étroite avec leur banque bénéficient probablement d'un taux d'intérêt faible.

Toutefois, en Tunisie, la réputation de l'entreprise reflétée à travers la relation client n'a aucun impact sur le taux d'intérêt exigé, dans le sens que les PME ne disposent pas d'un dossier qu'elles cherchent à préserver et à renforcer en honorant continuellement leurs engagements.

Dans une autre perspective, notre résultat remet en cause la notion de monopole de la banque ou encore le phénomène de la capture informationnelle qui se produit au profit du prêteur, puisque la corrélation entre la variable CLIENT et INTER est négative. Rajan (1992) et Sharpe (1990) ont avancé que la relation de clientèle permet à la banque de disposer d'informations sur l'entreprise, non accessibles aux autres banques. Si la firme choisit de changer de partenaire financier, cela peut être interprété par les autres banques comme un signe d'abandon de l'entreprise par le présent partenaire financier. La banque détient par conséquent un pouvoir de marché sur la firme qui lui permet de s'approprier une rente en pratiquant un taux d'intérêt plus élevé que celui qui pourrait être obtenu auprès des autres banques.

Par ailleurs, il semble que le taux d'intérêt ne soit pas tributaire du secteur d'activité (SECT.EC) des petites et moyennes entreprises constituant l'échantillon, ni de leur forme juridique (F.JURID). La non-significativité de ces deux paramètres semble logique puisque cette stratégie ne tient pas compte des caractéristiques inhérentes à chaque client, mais considère plutôt les entreprises solliciteuses de crédits comme un ensemble homogène lui permettant d'appliquer un contrat unique. Plus précisément, concernant la non-signification de la variable représentant la forme juridique de l'entreprise, il faut souligner les traits communs aux petites et moyennes entreprises tunisiennes. Il s'agit principalement de leur caractère familial (qualité des participants au capital social et recrutement du personnel) ainsi que de la fusion du patrimoine de l'entreprise à celui des propriétaires-dirigeants.

Le résultat trouvé s'oppose à celui de Bodt, Lobez et Statnik (2000) qui ont soutenu que ces deux paramètres (la forme juridique et le secteur d'activité) ne sont pas neutres et influencent le coût de crédit que supportent les entreprises et qui est pratiqué par une banque ayant adopté une stratégie de regroupement. Allessandrini (1998) a confirmé le rôle déterminant du secteur d'activité sur le taux d'intérêt, ajoutant que l'effet du cycle d'affaires à une influence prépondérante sur les taux d'intérêt à long terme.

Finalement, la stratégie de regroupement ne retient pas la spécificité de l'actif (SP. ACTF) comme un facteur significatif. Cela peut être expliqué par le fait que 
les petites et moyennes entreprises tunisiennes de notre échantillon ont des activités presque similaires ne nécessitant pas, par conséquent, un actif spécifique caractérisé par son non-redéploiement.

En retirant la variable CLIENT qui se révèle non significative dans la régression (1) en introduisant la variable LEVER, nous obtiendrons la formulation de la deuxième régression qui donne le modèle économétrique suivant :

INTER $_{i t}=a+b_{2}$ RISQ $_{i t, t+k}+c_{2}$ PLGR $_{i t}+d_{2}$ CASOL $_{i t}$ $+e_{2}$ NANTS $_{i t}+f_{2}$ HYPO $_{i t}+k_{2}$ LEVER $_{i t}+l_{2}$ F.JURID $_{i t}$ $+m_{2}$ SECT.EC $_{i t}+n_{2}$ SP.ACTF it $_{i t}$

\section{TABLEAU 4}

Estimations des paramètres relatifs au modèle (2)

Variable dépendante : taux d'intérêt (INTER)

\begin{tabular}{lccc}
\hline Variables & Coefficients & $\boldsymbol{t}$ & Signification \\
\hline RISQ & $-2,682 \mathrm{E}-02$ & $-1,013$ & 0,312 \\
[PLGAR] & $-0,119 \mathrm{E}-03$ & $-1,081$ & 0,279 \\
[CASOL] & $-0,948 \mathrm{E}-03$ & $-1,927$ & $0,054^{* *}$ \\
[NANTS] & $-0,352 \mathrm{E}-03$ & $-2,04$ & $0,041^{*}$ \\
[HYPOT] & $0,975 \mathrm{E}-03$ & 0,945 & 0,344 \\
LEVER & $0,988 \mathrm{E}-03$ & 1,985 & $0,047^{*}$ \\
[F.JURID= SA] & $-0,206$ & $-1,192$ & 0,234 \\
[SECT.EC= Industrie] & $-1,504 \mathrm{E}-02$ & $-0,589$ & 0,557 \\
SP.ACTF & 0,0645 & 0,625 & 0,531 \\
\hline
\end{tabular}

** Signification $<10 \% *$ Signification $<5 \%$.

$\mathrm{R}^{2}=0,508\left(\mathrm{R}^{2}\right.$ ajusté $\left.=0,437\right)$.

Les résultats de la régression révèlent que sur les neuf variables retenues, trois seulement sont significatives, à savoir le levier financier, la caution solidaire et le nantissement.

En effet, le risque demeure non significatif, ce qui concorde avec l'évidence appuyant la validité de la stratégie de regroupement et la neutralité du risque relatif à chaque entreprise sur la fixation du taux d'intérêt. Ainsi, nous pouvons présumer que la banque tunisienne, qui a affaire à une clientèle hétérogène, essaye de considérer le risque global du portefeuille des crédits. Elle offre ainsi un contrat homogène et unique, lui permettant d'économiser les coûts d'analyse afférents à l'étude des dossiers de crédit.

Parmi les variables relatives aux garanties exigées, nous trouvons que le contrat de crédit qui inclut la caution solidaire du dirigeant de l'entreprise ou un nantissement est caractérisé par une baisse du taux d'intérêt par rapport au contrat 
qui n'incorpore aucune garantie. Ce résultat rejoint l'effet incitatif ou révélateur des garanties dont la disponibilité est généralement combinée à une baisse du taux d'intérêt.

Outre ces modalités de garanties, le levier financier constitue un paramètre qui influence la détermination du taux d'intérêt chargé par la banque. Ce résultat rejoint l'énoncé de la théorie de signalisation voulant que la structure financière de l'entreprise soit un signal qui peut être interprété par la banque, dans un contexte d'asymétrie d'information, et la renseigner sur les perspectives financières du client. Ainsi, l'augmentation du levier financier signale un risque d'insolvabilité de l'emprunteur et se traduit par une augmentation du taux d'intérêt.

La corrélation positive entre le levier financier et le taux d'intérêt met en exergue un problème soulevé par Stiglitz et Weiss (1981). En effet, selon ces deux auteurs, l'augmentation du taux d'intérêt d'une manière prohibitive peut favoriser le risque moral de l'emprunteur et l'amener à adopter des comportements opportunistes.

Toutefois, à ce niveau, il est utile d'énoncer le rôle des règles prudentielles. En effet, en raison de l'absence de véritables investisseurs institutionnels en Tunisie, les banques concentrent l'effort le plus important dans le financement. Elles ont fait l'objet de plusieurs programmes de mise à niveau : renforcement des règles prudentielles en matière d'octroi de crédit et d'appréciation du risque, mise en place d'un ratio de liquidité, établissement de seuil de provisionnement pour assainir les bilans, etc.

Malgré les progrès effectués, certaines banques, notamment publiques, n'ont toujours pas accompli les efforts attendus et demeurent vulnérables à de possibles accidents conjoncturels. Pour autant, la veille assurée par la Banque centrale de Tunisie (BCT) permet de prévenir et d'accompagner par des mesures appropriées tout risque susceptibles d'émerger.

Finalement, nous remarquons qu'outre la modalité hypothèque (HYPOT) qui perd sa signifiance dans la deuxième régression, le secteur économique, la forme juridique, la modalité «plusieurs garanties » et la spécificité de l'actif n'ont aucune influence sur le taux d'intérêt accordé par la banque.

\section{Conclusion}

L'objectif de cette étude est de cerner la validité du regroupement des demandes de crédits des PME tunisiennes. Le contexte tunisien est caractérisé par une méfiance des banques commerciales à l'égard des petites et moyennes entreprises qui sollicitent du financement. La théorie relative au domaine bancaire a relevé 
que la fragilité du système informationnel de ces firmes, combinée aux carences managériales et aux mutations environnementales, sont les principaux facteurs expliquant la difficulté de définir le risque que présente chaque client potentiel.

Cette attitude frileuse des banques demeure vérifiable, malgré les enseignements de la théorie de l'agence, la théorie de signaux et la théorie relative aux mécanismes incitatifs. Devant l'accroissement de l'opacité informationnelle entre les PME et la banque, cette dernière doit opter pour une stratégie décisionnelle lors de l'accord de crédit, afin de minimiser son risque global, ce qui en outre lui permet de réaliser des économies d'échelle dans l'analyse des demandes de financement.

Nous constatons, après avoir mené cette étude, que la banque retenue adopte la stratégie de regroupement en matière d'octroi de crédits. Ce résultat soutient les caractéristiques que présente cette dernière en termes d'hétérogénéité de la clientèle composée des PME et la neutralité du facteur risque qui n'influe pas sur la détermination du taux d'intérêt.

Toutefois, les éléments qui peuvent amplifier ou influer sur le risque ne peuvent pas être déterminés convenablement et avec précision par la banque qui opte pour ce type de stratégie à dimension globale, à critère d'évaluation très restreint et à pouvoir explicatif faible.

La conception de cette stratégie se base sur une homogénéité absolue des entreprises quant à leur risque et perspective futures. Or, en réalité, cette supposition n'est pas vérifiable à cause des différences caractéristiques et particulières des clients qui poussent la banque commerciale tunisienne à les considérer en s'appuyant sur des éléments qui leur sont spécifiques.

Par ailleurs, cette stratégie peut augmenter aléatoirement le rationnement d'équilibre pour les entreprises tunisiennes dans le cas où, pour un taux d'intérêt donné, la demande serait supérieure à l'offre, la hausse du taux d'intérêt ayant alors un effet important sur la sélection défavorable. Dans cette situation, la banque n'opte pas pour le changement des conditions d'emprunt, et cela peut entraîner un rationnement durable du crédit susceptible d'affecter toutes les entreprises indépendamment de leur risque.

Ce rationnement de crédit peut être basé sur différents critères. En effet, la banque peut favoriser un ancien client et lui accorder les fonds demandés, bien qu'il présente un risque élevé, ou passe par une période de détresse financière. Ce scénario appuie le fait que le rationnement du crédit par les banques tunisiennes est essentiellement basé sur la relation de long terme ou de clientèle qu'elles entretiennent avec les PME. À ce niveau, il est évident qu'une inefficience dans l'allocation des crédits surgit et s'accentue s'il existe des liens de proximité informels entre le banquier et le client. 
Par conséquent, cette stratégie peut ne pas satisfaire le besoin de certaines banques commerciales à cause de sa passivité et de son pouvoir très réduit à cerner le risque réel auquel elles s'exposent par l'octroi de crédits.

Ainsi, des recherches ultérieures doivent vérifier s'il est possible de faire correspondre à la prise de décision d'octroi de crédits et à la politique de rationnement de la banque certains types d'information en fonction des éléments fournis par l'entreprise qui soumet une demande de crédit.

Dans une autre perspective, il serait utile d'évaluer dans quelle mesure ces inputs répondraient au développement d'une stratégie décisionnelle adaptée au contexte de la PME tunisienne. Par ailleurs, les études futures doivent se baser sur les informations figurant dans les contrats de crédits. En effet, le recours aux données comptables pour approximer les variables retenues entache les résultats d'une certaine imprécision. Plus explicitement, l'établissement d'une relation de clientèle entre une banque et une PME, souffrant de problèmes d'asymétrie d'information, favorise l'échange d'informations qualitatives et privées (caractère sérieux du dirigeant, son honnêteté, la confiance qu'il inspire au banquier, etc.). Ainsi, il serait pertinent d'intégrer dans les analyses ultérieures ces flux qui peuvent servir d'input dans la prise de décision concernant l'octroi de crédits.

\section{Bibliographie}

ALESSANDRINI, F. (1998), «Credit risk, interest rate risk, and the business cycle», Journal of Fixed Income, septembre, vol. 8, no 3, p. 42-53.

ANG, J.S. (1991), «Small business uniqueness and theory of financial management», Journal of Small Business Finance, vol. 2, no 3, p. 1-13.

AVERY, R.B., R.W. Bostic et K.A. SAMOLYK (1998), «The role of personal wealth in small business finance », Journal of Banking and Finance, vol. 22, n 1, p. 1019-1061.

BERGER, A. et G.F. UDELL (2002), «Small business credit availability and relationship lending: the importance of bank organisational structure », Economic Journal, vol. $95, n^{\circ} 2$, p. 319-325.

Berger, A., N.H. Miller, M.A. Petersen, R.G. Rajan et J.C. Stein (2002), «Does function follow organizational form? Evidence from the lending practices of large and small banks », Cahier de recherche, p. 19-25.

BERRYMANN, J. (1983), «Small business failure and bankruptcy : a survey of the literature», European Small Business Journal, vol. 1, nº 4, p. 47-59.

BESANKo, D. et A.V. THAKOR (1987), «Collateral and rationing: sorting equilibria in monopolistic and competitive credit market», International Economic Review, vol. 28, no 3, p. 671-690.

BESTER, H. (1985), « Screening vs. rationing in credit markets with imperfect information », American Economic Review, vol. 75, n $\mathrm{n}^{\mathrm{O}} 3$, p. 850-855.

Revue internationale P.M.E., vol. 17, nº 2, 2004 
BISSIRIOU, G. (1989), «La spécificité des besoins financiers des PME innovatrices », Revue française de gestion, vol. 73, $\mathrm{n}^{\circ}$ 1, p. 62-74.

Black, S. et P.E. STRAHAN (2001), «Entrepreneurship and bank credit availability », Cahier de recherche, p. 16-31.

BodT, E., F. LOBEZ et J.-C. STATNIK (2000), «Credit decision and adverse selection : an empirical study of banking behavior», Cahier de recherche, Université de Lille, Université du Littoral, p. 46-92.

BoLTON, J.E. (1971), «Report of the committee on liquidity of small firms », Journal of Political Economy, vol. 21, n 2, p. 102-125.

COLOT, V. et P.A. Michel (1996), «Vers une théorie financière adaptée aux PME: Réflexions sur une science en genèse », Revue internationale PME, vol. 9, $\mathrm{n}^{\circ} 1$, p. 141-166.

COSSIN, D. et T. HRICKO (2001), «Exploring the determinants of credit risk in credit default swap transaction data », Cahier de recherche, mai, Université HEC de Lausanne, p. 25-39.

DIAMOND, D.W. (1991), «Monitoring and reputation: the choice between bank loans and directly placed debt», Journal of Political Economy, août, vol. 99, n ${ }^{\circ} 4$, p. 689-721.

DUFFEE, G.R. (1998), « The relationship between treasury yields and corporate bond yield spreads », Journal of Finance, décembre, vol. 2, nº 6, p. 2225-2241.

ELSAS, R. et J.P. KRAHNEN (1998), «Is relationship lending special from credit-file data in Germany », Journal of Banking and Finance, vol. 22, n 2, p. 1283-1316.

FERNANDO, C. et R. MALLICK (2002), «The importance of being known : relationship banking and credit limits », Cahier de recherche, p. 13-16.

JAMES, C. (1987), «Some evidence on the uniqueness of bank bans, » Journal of Financial Economics, vol. 19, no 3, p. 217-235.

JENSEN, M.C. et H. MECKLING (1976), «Theory of the firm : managerial behaviour, agency costs and ownership structure», Journal of Financial Economics, vol. 3, n ${ }^{\circ} 2$, p. 305-360.

LEHMANN, E. et D. NEUbERGER (2002), « Do lending relationship matter ? Evidence from bank survey data in Germany», Cahier de recherche, Université de Konstanz et Université de Rostack, p. 45-59.

LELAND, H.E. et D.H. PYLE (1977), «Informational asymmetries, financial structure and financial intermediaries », Journal of Finance, vol. 32, n 5 , p. 371-387.

MERTON, R.C. (1974), «On the pricing of corporate debt: the risk structure of interest rates », Journal of Finance, vol. 29, n 4, p. 449-470.

MYARD, O. et B. ALPERTE (2003) «Le rôle des banques dans le financement de l'investissement », Marchés émergents, vol. 24, $\mathrm{n}^{\circ}$ 1, p. 2-3.

NAKAMURA, L. (1998), «Loan workouts and commercial bank information: Why banks are special», Cahier de recherche de la Banque fédérale de Philadelphia, p. $40-49$. 
OMri, A. et M. Bellouma (2003), «L'effet de la relation client sur la décision d'octroi de crédit dans le contexte tunisien », II ${ }^{\mathrm{e}}$ Conférence internationale de Finance, Tunisie, vol. 2, $\mathrm{n}^{\mathrm{0}} 2$, p. 5-9.

PEACOCK, R.W. (1985), «Finding the causes of small business failure», Cahier de recherche, Forum de management, vol. 11, n 2, p. 77-89.

PETERSEN, M. et R. RAJAN (1994), « The benefits of lending relationships : evidence from small business data, » Journal of Finance, vol. 49, n 1, p. 3-37.

PSILLAKI, M. (1995), «Rationnement du crédit et PME : une tentative de mise en relation », Revue internationale PME, vol. 8, $\mathrm{n}^{\text {os }} 3-4$, p. 67-90.

RAJAN, R.G. (1992), «Insiders and outsiders: the choice between informed and arm's length debt », Journal of Finance, vol. 47, n 2, p. 1367-1400.

Ross, S. (1977), «The determination of financial structure: the incentive signalling approach », Bell Journal of Economics, vol. 8, n 2, p. 23-40.

SCOTT, J.A. (2003), «Bank mergers and small firm financing », Journal of Money, Credit and Banking, vol. 35, $\mathrm{n}^{\circ}$ 2, p. 7-29.

SHARPE, S.A. (1990), «Asymmetric information, bank lending and implicit contracts : a stylized model of customer relationships », Journal of Finance, vol. 45, no 3 , p. 1069-1087.

STIGLITZ, J.E. et A. WeISS (1981), «Credit rationing in markets with imperfect information », American Economic Review, vol. 71, n 3, p. 393-410.

WTTERWULGHE, R. et F. JANSSEN (1995), «Freins et motivations de la cotation en Bourse des entreprises moyennes belges », $\mathrm{II}^{\mathrm{e}}$ Congrès international francophone des PME, p. 309- 325 . 